\title{
ESTIMATION DE LA DEFORESTATION DES FORETS HUMIDES A MADAGASCAR UTILISANT UNE CLASSIFICATION MULTIDATE D'IMAGES LANDSAT ENTRE 2005, 2010 et 2013
}

\author{
Rakotomala F.A. ${ }^{1,5}$, Rabenandrasana, J. C. ${ }^{2}$, Andriambahiny, J. E.4, Rajaonson, R. ${ }^{2}$, Andriamalala, F. ${ }^{4}$, Burren, $^{2}{ }^{2}$, \\ Rakotoarijaona, J.R. ${ }^{3}$, Parany, B.L.E. ${ }^{4}$, Vaudry, R. ${ }^{1}$, Rakotoniaina, S. ${ }^{5}$, Ranaivosoa, R. ${ }^{3}$, Rahagalala, P. ${ }^{3}$, Randrianary, T. \\ Grinand, C. ${ }^{6}$, \\ 1: Etc Terra, Lot VE 26 L, Ambanidia, 101 Antananarivo, Madagascar \\ 2: WCS, Wildlife Conservation Society, Villa Ifanomezana, Face II A78 D Soavimbahoaka, BP 8500 Antananarivo, \\ Madagascar \\ 3 : ONE, Office National de l'Environnement, Rue Rainilaiarivony, BP 822, Antananarivo, Madagascar \\ 4 : MNP, Madagascar National Parks, Madagascar, BP 1424, 103 Antananarivo, Madagascar \\ 5 : IOGA, Instituts et Observatoires de Géophysiques d'Antananarivo, Université d'Antananarivo, Faculté des sciences, \\ Madagascar \\ 6 : Etc Terra, 127 rue d'Avron, Paris - France
}

\begin{abstract}
Résumé
La spatialisation de la dynamique forestière est essentielle à l'estimation des niveaux d'émissions de gaz à effet de serre dans les forêts affectés par la déforestation. L'objectif de cette étude était d'estimer le niveau de la déforestation et de mieux comprendre la dynamique des forêts humides de l'Est de Madagascar entre 2005-2010-2013. Une approche de classification supervisée d'images Landsat multidates utilisant l'algorithme Random Forest a été utilisée. Une carte complète de l'évolution du couvert forestier à 30 mètres de résolution spatiale a été produite sur 20,5 millions d'hectares. Le résultat a été validé par photo-interprétation de près de 11000 points à partir d'un échantillonnage de points régulier et sur la base d'images satellites SPOT et Landsat pour la période 2010-2013. La précision globale de la carte de la déforestation ainsi produite a été évaluée à environ 90\%. La superficie des forêts humides de l'Est de Madagascar a ainsi été estimée à 4,5 millions d'hectares en 2005, 4,4 millions d'hectares en 2010 et 4,3 millions d'hectares en 2013. Le taux de déforestation annuel a augmenté de 0,5\%. à 0,9\% entre les deux périodes étudiées. Cette étude permet d'envisager le développement d'une démarche de suivi de l'état des forêts fiable, à moindre coût et reproductible dans le temps.
\end{abstract}

Mots-clés : Télédétection, images Landsat, RandomForest, déforestation, dynamique forestière.

\section{Abstract}

Monitoring forest state is required to estimate the levels of greenhouse gases emissions from deforestation. The objective of this study was to estimate deforestation trends for 2005-2010 and 2010-2013 periods in the eastern rainforests ecoregion of Madagascar. A supervised classification approach of multi-date Landsat images using the Random Forest algorithm was used. A complete $30 \mathrm{~m}$ map of the forest cover change was produced on 20.5 million hectares. The result was validated by photo interpretation of nearly 11000 points from a regular sampling grid on the basis of SPOTand Landsat satellite images acquired in 2010-2013. The overall mapping accuracy was estimated to be about $90 \%$. The area of Madagascar rainforests has been estimated at 4.5 million hectares in 2005, 4.4 million hectares in 2010 and 4.3 million hectares in 2013. The annual rate of deforestation has increased from $0.5 \%$. to $0.9 \%$ between the two periods. This study could be considered as a reliable cost-effective and reproductible approach for monitoring forests in Madagascar.

Keywords: Remote sensing, Landsat images, Random Forest, deforestation, forest cover change.

\section{Introduction}

La réduction du couvert forestier n'est pas un phénomène récent et est associée depuis longtemps aux activités humaines (Williams et al., 2002). À partir d'observations satellitaires, Achard et al., (2002) ont montré que les forêts couvraient 4 milliards d'hectares soit près de $30 \%$ de la surface émergée du globe en 2000. Plus récemment, Hansen et al., (2013) ont estimé que le couvert forestier mondial avait subi une perte de 2,5 millions de kilomètres carrés durant la période 2000 à 2012, soit un rythme de 2100 kilomètres carrés chaque année, principalement localisés en climat tropical. Par ailleurs, les écosystèmes forestiers constituent un réservoir terrestre important de carbone. La conversion de terres forestières en terres agricoles (déforestation) est responsable de $17 \%$ des émissions annuelles de Gaz à Effet de Serre (GES) d'origine 
anthropique (GIEC, 2007). Afin d'apporter des solutions pour réduire le rythme actuel de la déforestation, le mécanisme REDD+ (Réduction des Émissions de gaz à effet de serre dues à la Dégradation et à la Déforestation, augmentation des stocks et gestion durable des forêts) a été introduit lors de la $13^{\text {ième }}$ session de la Conférence des Parties en 2007 à Bali suite à des intenses négociations. Ce mécanisme implique le suivi de l'évolution des terres forestières afin de mesurer et vérifier les politiques, programmes et projets visant à conserver ou augmenter cette ressource (Verchot et al., 2009). Les données et outils de télédétection sont indispensables pour atteindre ces objectifs (DeFries et al., 2005; Gibbs et al., 2007). Cependant malgré l'existence de lignes directrices (GOFC-GOLD, 2014; GIEC, 2006), les techniques de télédétection doivent être améliorées afin de permettre de surveiller des grandes surfaces forestières, d'une réduction de la fréquence de mesure et améliorer le niveau de précision sur les parcelles (patchs) de déforestation.

Madagascar constitue des centres de biodiversité le plus importants du monde (Myers et al., 2000), où les forêts tropicales humides couvraient 11,5 millions d'hectares dans les années 1950 (Harper et al., 2007). Néanmoins, d'importantes menaces pèsent sur la flore, notamment par la destruction de l'habitat pour la pratique de «tavy » (culture sur brulis).

Dans le cadre de la mise en œuvre de sa stratégie REDD+ pour minimiser ces menaces, Madagascar doit se doter d'estimations récentes de son couvert forestier et proposer des outils de mesure et suivi reproductible, fiable et efficient (GOFC-GOLD, 2014). L'écorégion des forêts humides a été identifiée comme prioritaire pour l'estimation de ses taux de déforestation et de ses stocks importants de carbone (RPP, 2014). Cette zone concentre également les principaux défis en termes de suivi de la déforestation par télédétection (relief, nuage, saisonnalité, étendue).

Ainsi, en 1990, le taux de la déforestation atteignait un niveau alarmant de $1 \%$ par an et les forêts humides ne couvraient plus que 5,6 millions d'hectares (Harper et al., 2007). Ces chiffres ont été actualisés pour les périodes 1990 -2005 (MEFT-Cl, 2009) et $2005-2010$ (MEFT-ONE-Cl, 2013) à partir des analyses basées sur des images Landsat. Ces études ont montré que les surfaces des forêts humides diminuent entre les années 1990 - 2010 et le taux de déforestation montrait une légère baisse, passant de $0,8 \%$ par an entre 1990-2000 à 0,5\% par an pour la période 20002010. Ces études présentent toutefois deux limites importantes: i) la forte présence de nuages sur les images utilisées ne permet pas une information exhaustive et ii) l'Unité Minimum de Cartographie (UMC) définie à 1 hectare (incluant les forêts et déforestation) exclut les petites parcelles de défrichement.

En 2012, la carte de déforestation mondiale produite par l'Université du Maryland (Hansen et al., 2013) a attiré une grande attention des scientifiques.
Cependant, Tropek et al.,(2014) ont montré que cette carte surestime la couverture forestière et sous-estime le taux de déforestation au niveau local.

Le suivi de la déforestation des forêts humides à Madagascar par télédétection doit faire face à plusieurs défis. Les forêts se trouvent dans les régions montagneuses à l'Est de l'île sur les reliefs très accidentées, seul $5 \%$ de la superficie totale est plane (Rakotomalala et al., 2012). Les parcelles de tavy sont généralement localisées sur les versants de faibles altitudes et difficiles à détecter avec les images satellites à cause des effets de reliefs sur ces images (Vieilledent et al., 2013). Par ailleurs, les parcelles de défrichement ou de déforestation sont de petites tailles $(0,6$ hectare en moyenne (Grinand et al., 2013)) compte tenu des fortes pentes et des difficultés d'accès. Ceci explique qu'avec les images Landsat actuellement disponibles pour ce type d'étude, il est difficile de détecter les parcelles de déforestation. Les parcelles de défrichement-brûlis se situant souvent à proximité l'une de l'autre spatialement, il faut que l'intervalle de temps soit suffisamment long pour que les petites surfaces concernées par la déforestation soient détectables. De plus, cette région est sujette à des fortes couvertures nuageuses limitant l'application de la télédétection optique pour l'analyse de l'évolution de la déforestation. Enfin, la vaste étendue de la zone d'étude demande à traiter un grand nombre d'images avec des dates d'acquisition différentes. Ceci pose des problèmes temporels car les saisons ont un impact fort en termes de phénologie et densité du couvert végétal. Une étude récente sur des sites pilotes REDD+ à Madagascar (Grinand et al., 2013) a proposé des innovations méthodologiques i) pour la classification des images afin de gérer les effets de saisonnalité sur les images à classifier (approche multidate) et ii) pour le posttraitement afin de réduire la surface minimale détectée pour les parcelles de déforestation à 0,36 hectare tout en produisant une qualité de classification satisfaisante. Cependant cette étude pilote n'a pas été testée à une échelle nationale, ni sur des intervalles de temps de moins de 5 années.

L'objectif de cette étude a été de produire une carte du couvert forestier actualisée entre 2005 - 2010 - 2013 et évaluer la déforestation à l'échelle de l'écorégion des forêts humides de Madagascar. L'approche utilisée par Grinand et al., (2013) a été adaptée pour de grandes étendues et pour des intervalles de temps réduits (Tableau 1). La carte finale de la déforestation a été validée à l'aide d'une photo-interprétation sur des images à haute résolution spatiale (SPOT $5-10$ mètres) pour la période 2010 - 2013 (Corbane et al., 2015).

\section{Zone d'étude}

Madagascar est une île d'une superficie de 590000 $\mathrm{km}^{2}$, situés dans l'Océan Indien et est caractérisée par quatre grands types d'écosystèmes forestiers définis sur les bases des différentes subdivisions 
phytogéographiques de l'Inventaire Écologie et Floristique Nationale (Humbert et al., 1965): les forêts humides de l'est, les forêts sèches de l'ouest, les forêts épineuses du sud et enfin les mangroves (figure 1).

La présente étude concerne les forêts humides dont la définition officielle est de minimum $30 \%$ de couvert forestier, une hauteur de plus de $5 \mathrm{~m}$ et sur plus de 1 ha. Elle se situe sur le versant oriental du nord au sud de la grande île. Deux types de climat coexistent sur cette région (Cornet et al., 1974): le climat de type perhumide, à une altitude inférieure à $800 \mathrm{~m}$ et le climat de type humide, au-dessus de $800 \mathrm{~m}$ mais avec les sous-domaines écologiques suivants (White et al., 1983):

- domaines de l'est et du Sambirano $(0-800$ $\mathrm{m})$ : forêts denses humides sempervirentes de basse altitude et forêts littorales ;

- domaines du centre versants orientaux (800 - $1800 \mathrm{~m})$ : forêts denses humides sempervirentes de moyenne altitude ;

- domaines du centre étage des montagnes (> $1800 \mathrm{~m})$ : forêts sclérophylles de montagne et fourrés sclérophylles de montagne ;

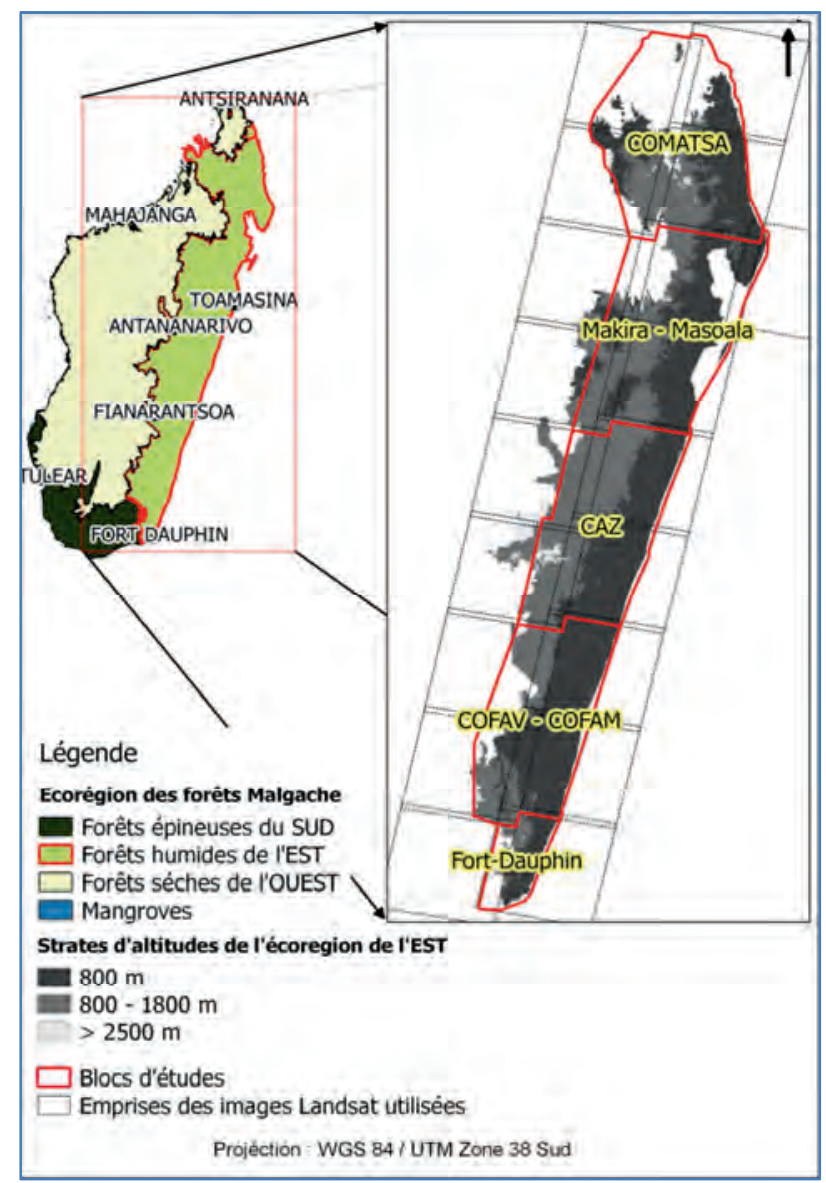

Figure 1: Localisation de la zone d'étude, des emprises d'images Landsat et des blocs utilisés pour la photo-interprétation et le traitement des données. Les 5 blocs sont du nord au sud: Comatsa, Makira-Masoala, Caz, Cofav-Cofam et Fort-Dauphin.

\section{Matériel et Méthodes}

L'analyse multidate d'images satellites a été utilisée dans cette étude en considérant l'approche « Wall-toWall » (GOFC-GOLD, 2014). La première étape consiste à la collecte et à la correction géométrique des images satellites. Des calculs des indices géomorphologiques et radiométriques ont été réalisés suivis de la délimitation des parcelles d'entrainement pour la classification supervisée pixel à pixel basée sur l'algorithme RandomForest. Deux procédures de validation ont été mise en place: i) «validation interne " lors de la phase d'apprentissage de l'algorithme et ii) «validation externe » à partir de la photo-interprétation d'un grand nombre de points sur un maillage régulier et différentes sources de données spatiales (données d'Hansen, SPOT - 5 et Landsat). Ces étapes sont présentées dans les paragraphes suivants.

\subsection{Choix et collecte des images de référence}

Les images Landsat utilisées dans cette étude ont été téléchargées dans les archives de l'USGS (http://earthexplorer.usgs.gov et http://glovis.usgs.gov). Les images ont été sélectionnées de façon à ce qu'elles aient moins de $20 \%$ de couverture nuageuse sur la totalité de la scène et moins d'un pixel de décalage spatial pour chaque année de références: 2005, 2010 et 2013. Les produits «Global Land Survey " (GLS) de Landsat 5 (pour 2005 - 2010) et L1T de Landsat 8 (pour 2013) ont été choisis afin d'obtenir des données corrigées du point de vue géométrique et radiométrique. En effet, d'après Gutman et al., (2008), ces données disposent des qualités radiométriques et géométriques satisfaisantes pour effectuer des analyses de changement d'occupation du sol et notamment l'analyse historique de déforestation. Toutefois, le choix des produits GLS a été limité à cause de la couverture nuageuse sur quelques images. Dans certains cas, elles ont été remplacées par les images du satellite Landsat 7. Cependant, du fait d'une défaillance du capteur (Scan Line Corrector ou SLC) depuis 2003, les images Landsat 7 des années 2005 et 2010 présentent quelques centaines de mètres voire quelques kilomètres d'information manquante (stripping) même si elles ont de bonnes qualités géométrique et radiométrique (Barsi et al., 2007). De ce fait, plusieurs images d'une même année de référence ont été fusionnées afin de combler ce défaut. Pour assurer la disponibilité et la bonne qualité des images, les dates d'acquisition ont été choisies à une année près de la référence (tableau 1).

Afin de faciliter les traitements de ces données, l'écorégion a été subdivisée en cinq blocs pour que quatre équipes travaillant dans ce projet puissent se partager le travail. Chaque bloc est constitué d'un maximum de cinq scènes d'images pour alléger le processus de traitement (figure 1). 


\subsection{Prétraitements}

Les bandes de toutes les images téléchargées ont été compilées (stacking) en un seul fichier constituant ainsi une image multidate. Préalablement, une vérification de la précision géométrique a été faite visuellement sous ENVI et QGIS, dans le cas où un décalage supérieur à un pixel est observé, une rectification d'image à image a été réalisée en prenant comme image de référence les images GLS 2005. Les images ont été mosaïquées pour chaque année de référence et par blocs d'étude.
Ensuite, les mosaïques ont été projetées dans le système de projection : WGS 84 - UTM zones 38 Sud. Les scènes avec une faible couverture nuageuse ont été systématiquement mises au-dessus de celles qui présentent de nébulosité plus forte afin de minimiser la couverture nuageuse sur la carte finale.

Les décalages radiométriques dus aux différences des dates d'acquisition ont été minimisées en faisant une égalisation d'histogramme tout en prenant les images les plus nettes comme références.

\begin{tabular}{lllllll}
\hline Blocs d'étude & $\begin{array}{c}\text { Références } \\
\text { des } \\
\text { scènes }\end{array}$ & \multicolumn{2}{c}{ Années de référence } & \multicolumn{2}{l}{$\begin{array}{c}\text { Intervalle de temps } \\
\text { (en années) }\end{array}$} \\
& $158-069$ & $02 / 06 / 2004$ & $09 / 08 / 2008$ & $28 / 12 / 2013$ & 4,19 & 5,39 \\
& $159-069$ & $18 / 08 / 2006$ & $10 / 06 / 2010$ & $26 / 06 / 2013$ & 3,81 & 3,05 \\
Comatsa & $158-070$ & $27 / 08 / 2006$ & $05 / 10 / 2010$ & $19 / 06 / 2013$ & 4,11 & 2,71 \\
& $159-070$ & $25 / 04 / 2005$ & $10 / 06 / 2010$ & $07 / 09 / 2013$ & 5,13 & 3,25 \\
\hline \multirow{4}{*}{ Makira - Masola } & $157-071$ & $25 / 03 / 2005$ & $04 / 11 / 2011$ & $07 / 02 / 2014$ & 6,62 & 2,26 \\
& $158-071$ & $27 / 01 / 2005$ & $20 / 05 / 2011$ & $18 / 03 / 2014$ & 6,31 & 2,83 \\
& $159-071$ & $06 / 02 / 2006$ & $05 / 05 / 2009$ & $13 / 08 / 2013$ & 3,24 & 4,28 \\
& $158-072$ & $10 / 06 / 2007$ & $18 / 01 / 2011$ & $18 / 03 / 2014$ & 3,61 & 3,16 \\
Caz & $159-072$ & $20 / 03 / 2004$ & $30 / 04 / 2010$ & $13 / 08 / 2013$ & 6,12 & 3,29 \\
\hline Cofav- & $158-073$ & $11 / 06 / 2007$ & $08 / 07 / 2011$ & $18 / 05 / 2013$ & 4,08 & 1,86 \\
Cofam & $159-073$ & $28 / 04 / 2006$ & $01 / 05 / 2010$ & $25 / 05 / 2013$ & 4,01 & 3,07 \\
& $158-074$ & $24 / 06 / 2006$ & $08 / 07 / 2011$ & $18 / 05 / 2013$ & 5,04 & 1,86 \\
& $159-074$ & $25 / 04 / 2005$ & $07 / 06 / 2009$ & $25 / 05 / 2013$ & 4,12 & 3,97 \\
\hline Fort-dauphin & $158-075$ & $26 / 04 / 2005$ & $15 / 11 / 2009$ & $05 / 07 / 2013$ & 4,56 & 3,64 \\
\hline & $159-075$ & $11 / 09 / 2006$ & $30 / 03 / 2010$ & $23 / 04 / 2013$ & 3,55 & 3,07 \\
\hline & $158-079$ & $27 / 09 / 2006$ & $09 / 11 / 2010$ & $13 / 08 / 2013$ & 4,12 & 2,76 \\
& $159-076$ & $09 / 03 / 2005$ & $15 / 11 / 2009$ & $13 / 08 / 2013$ & 4,69 & 3,75 \\
\hline
\end{tabular}

Tableau 1. Base de données des images Landsat utilisées pour l'analyse historique de la déforestation entre 2005-2010 et 2010-2013. 11: Intervalle en nombre d'années décimales pour la période $2005-2010$ et 12 : Intervalle en nombre d'années décimales pour la période 2010 -2013.

\subsection{Classification supervisée multidate}

Dans la littérature scientifique, l'analyse bitemporelle est souvent adoptée pour la classification de la dynamique d'occupation du sol (Eastman et al., 2009). Cependant lorsque deux cartes d'occupation du sol correspondant à deux dates différentes sont combinées, les erreurs individuelles sont multipliées si l'on considère que les erreurs des deux cartes sont indépendantes (Fuller et al., 2003). Ainsi l'analyse multidate réduit les erreurs de classification par rapport à la comparaison de classifications produites pour plusieurs dates (GOFC-GOLD, 2014). Ceci nous a conduits à effectuer une classification supervisée sur des images multidates dans toute l'écorégion.

\subsubsection{Définition des classes d'occupation des sols}

Les classes d'occupation des sols et d'évolutions retenues se basent sur les recommandations de I'IPCC (2006) et des diverses études d'occupation du sol connues dans l'ensemble de l'écorégion (tableau 2). 


\begin{tabular}{|c|c|}
\hline $\begin{array}{l}\text { Typologies des parcelles } \\
\text { d'entrainement }\end{array}$ & Descriptions des classes de changement d'occupation du sol \\
\hline FFF & $\begin{array}{l}\text { Forêts « stables » entre } 2005 \text { et } 2013 \text { :Terres forestières humides } \\
\text { intactes entre } 2005 \text { et } 2013\end{array}$ \\
\hline FFP & $\begin{array}{l}\text { Déforestation entre } 2010 \text { et } 2013 \text { : Terres forestières humides } \\
\text { converties en Prairies/Terres cultivées entre } 2010-2013\end{array}$ \\
\hline FPP & $\begin{array}{l}\text { Déforestation entre } 2005 \text { et } 2010 \text { : Terres forestières humides } \\
\text { converties en Prairies/Terres cultivées entre } 2005-2010\end{array}$ \\
\hline FNN & Terres forestières en 2005 et Nuage/Ombre en 2010 et 2013 \\
\hline FFN & Terres forestières en 2010 et Nuage/Ombre en 2013 \\
\hline PPP & Prairies/Terres cultivées depuis 2005 \\
\hline $\mathrm{HHH}$ & Zones humides inchangées \\
\hline AAA & Sols nus/sables/roches inchangés \\
\hline MMM & Mangroves inchangées \\
\hline
\end{tabular}

Tableau 2.Typologie des classes d'évolution d'occupation du sol retenue pour la cartographie de la déforestation utilisé pour la photo-interprétation.

\subsubsection{Délimitation des parcelles d'entrainement}

L'approche de classification retenue étant une approche supervisée (cf 3.3.3), des parcelles d'entrainement sont nécessaires pour calibrer l'algorithme de classification. Elles ont été délimitées par photo-interprétation sur les images à classifier. Une composition colorée multidate de la bande spectrale rouge (bande numéro 3 de l'image Landsat) a été produite pour visualiser les différents typologies recherchés. Les parcelles ont été localisées en « grappes » (figure 2), c'est-à-dire par regroupement de plusieurs parcelles d'entrainement dans une même unité de paysage, ceci pour minimiser les confusions entre les classes de changements en délimitant bien les limites entre les typologies voisines.

L'échantillonnage des parcelles d'entrainement a été fait de façon raisonné en valorisant surtout la connaissance sur terrain des équipes. Les grappes ont été réparties sur l'ensemble de l'image à classifier. Les contours ont été affinés et soigneusement vérifiés en les superposant sur les images satellites à très haute résolution disponibles dans Google Earth (Quickbird). Au final, les résultats de la photo-interprétation pour les trois dates étudiées ont été stockés dans une base de données géolocalisée (BDD parcelle d'entrainement (PE) au format shapefile) regroupant 2700 polygones de 24000 hectares au total. La taille médiane des polygones liés à la déforestation entre 2005 - 2010 (FPP) et $2010-2013$ (FFP) est de 1,96 hectare et 1,9 hectare respectivement. Ces valeurs permettent de quantifier les surfaces moyennes défrichées annuellement en les divisant par les intervalles moyens présentés dans le tableau 1. Ce qui donne environ 0,5 hectare de taille moyenne d'une parcelle de tavy. Ce chiffre est comparable aux 0,6 hectare mesurés par Grinand et al., (2013). Sur le terrain, il est probable que cette surface moyenne soit plus élevée si on tient en compte la pente, et qu'elle soit variable selon les régions (organisation individuelle ou collective pour le tavy, mécanisation, etc.). La surface de défrichement annuelle est donc similaire à l'UMC.

\subsubsection{Classification}

L'algorithme RandomForest (RF), développé par Breiman et al., (2001), a été sélectionné pour ses bonnes capacités prédictives de l'occupation du sol (Gislason et al., 2006) et dans le cas d'analyse temporelle (Schneider et al., 2012).

C'est un algorithme de classification supervisée non paramétrique qui combine l'algorithme d'arbres de décision et une technique d'agrégation (bagging). II est présent dans package "randomForest " (Liaw et al., 2002) du logiciel R. L'algorithme construit une multitude d'arbres de décision par tirage aléatoire d'observation (pixels) et de variables. L'affectation de la classe finale est réalisée par vote majoritaire à partir de l'ensemble des arbres.

Le choix des variables explicatives influe sur la qualité du modèle de classification. Afin d'améliorer la discrimination des classes étudiées, plusieurs indices dérivés des canaux primaires des images satellites ont été calculés en plus des variables radiométriques, notamment l'indice normalisé de végétation (NDVI, Rouse et al., 1974), l'indice de réflectance du proche infrarouge (NIRI) et l'indice de teneur en eau par différence normalisée (NDWI, Gao et al., 1996). En plus de ces indices radiométriques, des indices géomorphologiques (l'altitude, la pente et la rugosité) dérivés d'un Modèle Numérique de Terrain à 30 mètres de résolution ont été introduits dans le but d'atténuer l'effet de relief sur les réflectances des canaux primaires (Tachikawa et al., 2011).

Ainsi, plusieurs modèles avec différentes combinaisons de variables ont été testés afin de déterminer la meilleure combinaison de variables pour la détection de la déforestation. Le choix de la meilleure combinaison s'est appuyé sur des indicateurs de performance lors de la validation interne décrite ci-après 

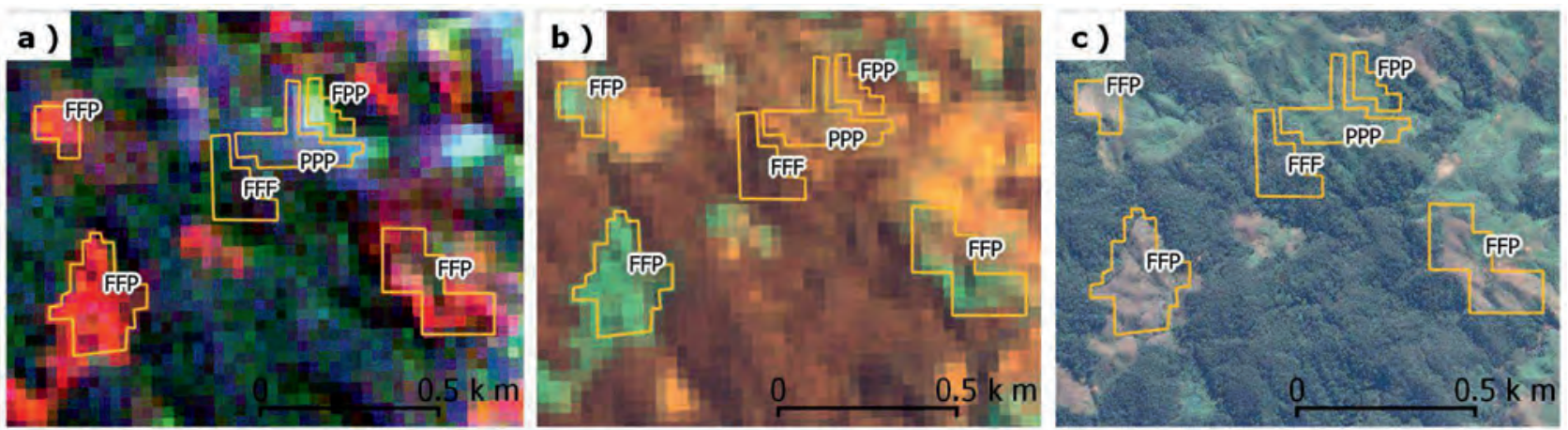

Figure 2 : illustration du processus de photo-interprétation lors du choix des parcelles d'apprentissages.

a) Mise en évidence des changements de classes sur une composition colorée multidates des images Landsat: (R: Band3-2013; G: Band3-2010; B: Band3-2005). En vert clair, les déforestations entre 2005et 2010 (FPP), en rouge, les déforestations entre 2010 et 2013 (FFP) et en vert sombre, les forêts « stables » depuis 2005 (FFF).

b) Délimitation des classes sur l'image Landsat 2013 à classifier (composition colorée : R: Band4; G: Band5; B: Band3). En orange foncé, les forêts, en orange clair les anciennes parcelles de déforestation (entre 2005 et 2010) et en cyan les nouvelles parcelles de déforestation observées sur l'image 2013;

c) Vérification et affinement sur Google Earth (décembre 2014), type d'image : satellite Quickbird (50 centimètres de résolution).

\subsubsection{Validation interne de la classification}

Le modèle a été construit en gardant les paramètres par défaut de l'algorithme RF, certains auteurs ayant montré qu'ils produisaient les meilleurs résultats (Rodriguez-Galiano, 2012). RF utilise les 2/3 (70\%) du jeu de données d'apprentissage pour la calibration et les $1 / 3(30 \%)$ restant pour la validation (production de la matrice de confusion et indicateurs de qualité). Sur la base de ces placettes de validation (30\%), l'algorithme calcule un indicateur de performance du modèle, appelé erreur Out-Of-the Bag (OBB) (Bylander et al., 2002). Plus cette erreur tend vers zéro, plus le modèle est bon. Outre cet indicateur, une matrice de confusion est produite pour évaluer la qualité " intrinsèque » du modèle. Elle permet de calculer les pourcentages d'erreur d'omission et de commission entre les diverses classes ainsi que l'indice Kappa (Cohen et al., 1960 et Caloz et al., 2001). Le modèle correspondant aux meilleurs indicateurs de qualité a été utilisé pour la classification de l'ensemble du bloc d'étude.

\subsection{Post-classification}

Une étape de post-classification a été implémentée afin de nettoyer l'image de classification brute, la faire correspondre aux exigences d'UMC et réduire les erreurs. Ainsi, un filtre convolutif majoritaire de dimension $3 \times 3$ pixels a été utilisé. Ensuite, deux filtres majoritaires ont été appliqués consécutivement afin d'éliminer les "parcelles de forêt" inférieure à 1 hectare (soit $3 \times 3$ pixels sur une image Landsat) et les parcelles de déforestation $(0,36$ hectare soit $2 \times 2$ pixels).

Pour disposer d'une cartographie complète de l'écorégion sans nuages, nous nous sommes appuyés sur une source extérieure notamment les données issues de l'étude de Hansen et al., (2013). Le pourcentage de couverture forestière en 2000 (treecover) ainsi que les pixels déforestés par année (lossyear) pour la période 2000 à 2012 produite par ces auteurs sont disponibles sur internet. Un seuil de $75 \%$ de couverture forestière a été appliqué pour estimer l'étendue forestière en 2000. Une carte de déforestation pour les périodes 2005 - 2010 et 2010 - 2012 a ensuite été créée à partir des pixels classés « déforestation». En superposant la carte de déforestation produite dans le cadre de cette étude avec celle dérivée d'Hansen et al., (2013), il a été possible d'éliminer les pixels identifiés comme ombres et nuages. Cette étape est résumée dans la figure 3.
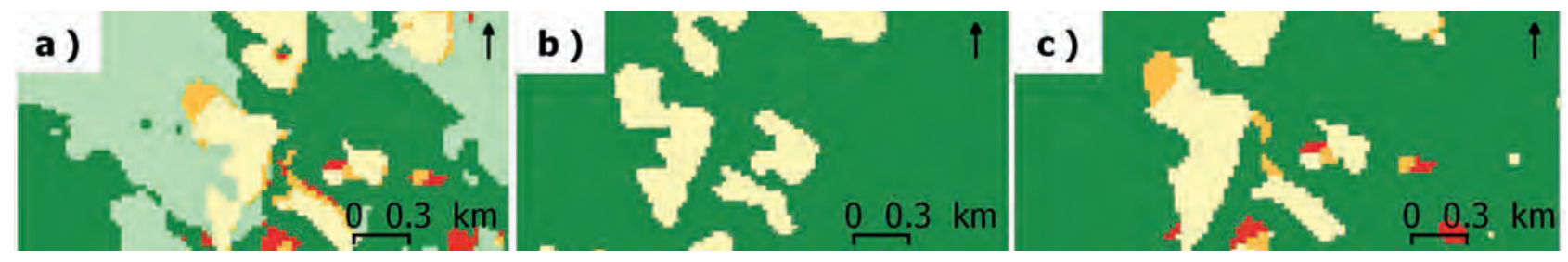

Figure 3. a) : Résultat de classification après filtrages avec les classes nuage et ombre (en vert clair) ; b) Carte forestière dérivée des données de Hansen et al., (2013); c) Combinaison de a) et b) pour produire la carte finale sans nuages entre 2005 et 2013 du PERR-FH (en vert les forêts intactes en 2013, en orange la déforestation entre 2005 2010 et en rouge la déforestation entre 2010 - 2013). 


\subsection{Validation externe des résultats}

Cette étape consiste en une confrontation statistique du résultat de la classification précédente (issue des images LANDSAT) avec le résultat de l'observation d'images satellites sur des points d'échantillonnage (approche "point sampling ») et pour deux dates historiques (2010 et 2013). Le choix de la localisation des points et les images à photo-interpréter ont été définis de manière à être complètement indépendants de la carte à valider.

L'échantillonnage de points a été réalisé sur des images à haute résolution: SPOT 5 de 10 mètres de résolution et sur des images LANDSAT de 30 mètres de résolution. Les images SPOT ont été commandées et collectées via la station "Surveillance de l'Environnement assisté par Satellite - Océan Indien» (SEAS-OI) pour la plupart des cas et dans certains cas à partir de la base de données image Planet Action. Au final, quatre régions sont couvertes par ces images à haute résolution et pour deux dates historiques (figure 4).

Une carte des pertes forestières a été produite sur la base des données de Hansen et al., (2013). Un échantillonnage régulier sur une grille carrée de $20 \mathrm{~km}$ x $20 \mathrm{~km}$ a ensuite été réalisé entre $2005-2012$ afin d'identifier les régions à forte pression de déforestation (hot spot). Pour se focaliser sur ces zones, la carte a été subdivisée en trois catégories selon l'intensité de la déforestation (tableau 3).

\begin{tabular}{ccc}
\hline $\begin{array}{c}\text { Intensité de la déforestation [ha/an] } \\
\text { d'après Hansen et al (2013) }\end{array}$ & $\begin{array}{c}\text { Niveau de pression de la } \\
\text { déforestation }\end{array}$ & $\begin{array}{c}\text { Espacement du } \\
\text { semis de point [km] }\end{array}$ \\
\hline$<20$ & Faible & $5 \times 5$ \\
$20-50$ & Moyenne & $1 \times 1$ \\
$>50$ & Forte & $0,5 \times 0,5$ \\
\hline
\end{tabular}

Tableau 3: Espacement du semis de points régulier utilisé pour la validation externe selon la pression de la déforestation issue des données d'Hansen et al., (2013).

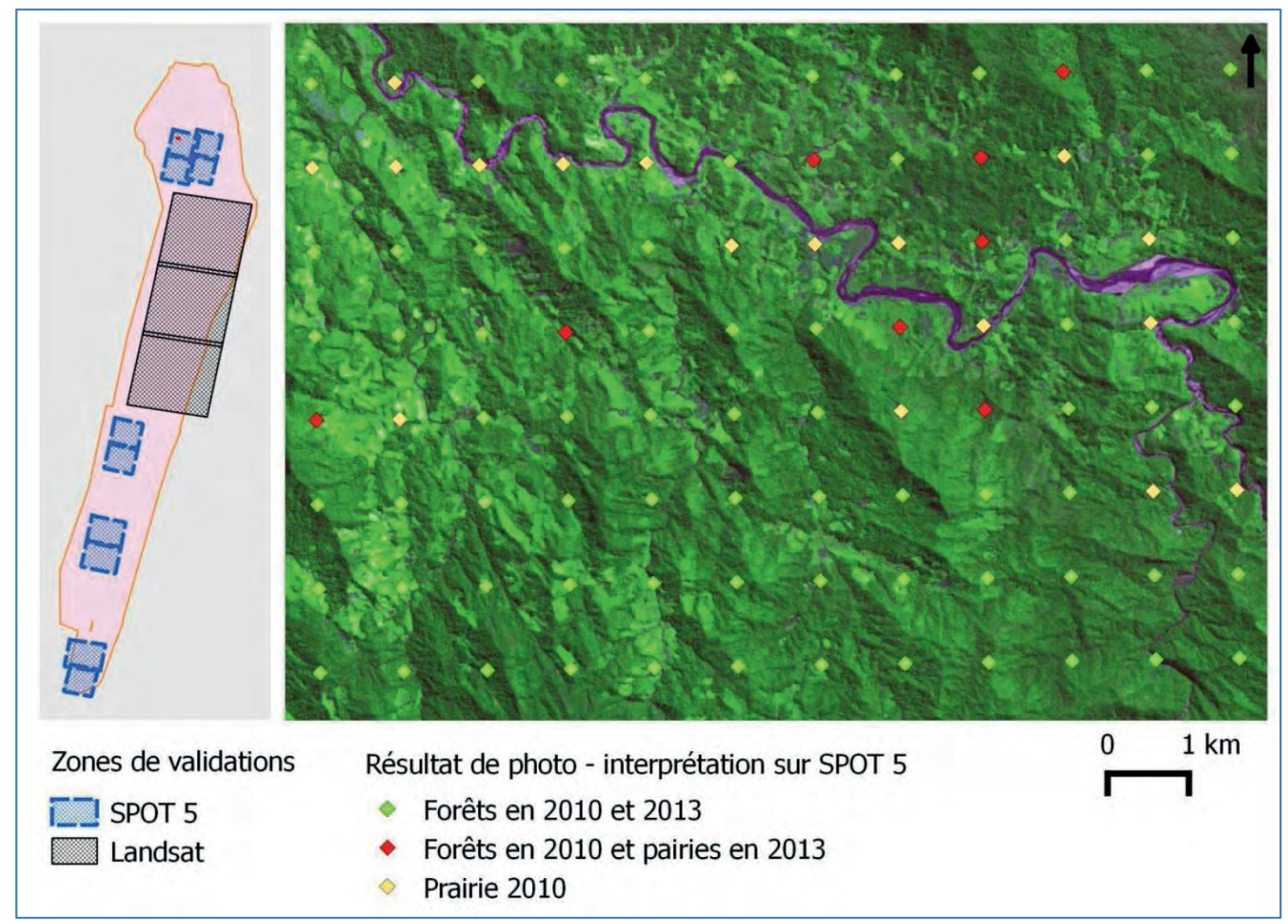

Figure 4. Illustration du processus de validation externe. À gauche : zones de validation et à droite, extrait d'une grille d'échantillonnage (1 kilomètre) sur des images SPOT 5 pour le bloc Comatsa. (Images distribuées par SEAS-OI @ CNES. Distribution Astrium Services / Spot image S.A., France, tous droits réservés). 


\subsection{Calcul du taux de déforestation}

Le taux de déforestation annuel résulte du rapport entre la surface défrichée sur une période, divisée par la surface forestière initiale et par le nombre d'années de la période (Menon et al., 1997, Ramesh et al., 1997, Narendra Prasad et al., 1998). Cependant, plusieurs publications ont récemment mis en lumière que l'évolution de la perte forestière ne peut pas être obtenue avec ce simple rapport (Puyravaud et al., 2002). La formule standardisée proposée par Puyravaud et al., (2002) a été adoptée pour calculer le taux de déforestation annuel pour cette étude (équation 1).

$$
\theta=-\frac{1}{t_{2}-t_{1}} \ln \left(\frac{A_{2}}{A_{1}}\right) * 100
$$

Avec

$A 1=$ superficie forestière de l'année initiale

A2 = superficie forestière de l'année finale

t1 = date d'acquisition exacte de l'image pour l'année initiale

t2= date d'acquisition exacte de l'image pour l'année finale

Ce mode de calcul demande de connaitre exactement l'intervalle entre les deux dates (t1 et t2). Comme on l'a vu précédemment (tableau 1), cette étude historique de la déforestation, comme toute autre étude, n'utilise pas une seule date (jour dans l'année) par année pivot, il existe des intervalles de temps variables selon les scènes dont il faut prendre en compte. Pour calculer le taux annuel de déforestation d'une région couverte par plusieurs $(N)$ scènes, la méthode adoptée a été de calculer la moyenne pondérée des taux de déforestation par scène (équation 2) afin de palier au problème lié à l'intervalle de temps qui n'est pas toujours le même pour chaque scène d'images (tableau 1). Les coefficients de pondération ont été obtenus en faisant le rapport de la surface de la forêt sur la scène concernée avec la surface totale de la forêt sur la région concernée (équation 3 ).

$$
\begin{gathered}
\theta_{r}=\frac{1}{S_{T}} \sum_{i=1}^{N} \omega_{i} \cdot \theta_{i}(2) \\
\operatorname{Avec} \omega_{i}=\frac{S_{i}}{S_{T}}(3)
\end{gathered}
$$

Où :

$\theta_{i}:$ Taux de déforestation dans une scène « i »appartenant à la zone concernée

$\theta_{r}$ : Taux de déforestation d'une région

$S_{i}$ : Surface totale de la forêt dans une scène

$S_{T}$ : Surface totale de la forêt dans la région

$\omega_{i}$ : Coefficients de pondération

\section{Résultats}

\subsection{Choix du modèle et validation interne}

Les résultats des différents tests effectués sur trois familles de variables avec cinq combinaisons différentes sont présentés dans le tableau 4. Ce test a été réalisé sur le bloc Comatsa avec 493 parcelles d'entrainement. On observe qu'en utilisant les 6 bandes spectrales des images satellitaires (modèle 1), l'OOB du modèle est estimé à $3,86 \%$. Lorsque l'on ajoute les indices d'occupations du sol (NDVI, NIRI et NDWI) avec ces bandes spectrales (modèle 2), le modèle s'améliore, mais très légèrement, avec une $\mathrm{OOB}$ de $3,45 \%$. Ainsi le fait d'ajouter ces néocanaux n'apporte pas plus d'information dans le modèle. Inversement, le fait d'ajouter des variables géomorphologiques améliore considérablement le modèle (modèle 3), avec une erreur $\mathrm{OOB}$ inférieure à 3 . Par ailleurs, le fait d'enlever les bandes 1 et 2 et de rajouter les indices d'occupations du sol a augmenté l'erreur dans le modèle 4.

Au final, le modèle 5 a été retenu pour l'ensemble des blocs puisqu'il intègre les indices d'occupation du sol parmi les covariables alors que le modèle 3 , malgré ses indices de qualité très légèrement supérieurs, est fortement influencé par l'importance des variables géomorphologiques. Les indices de précision des modèles de classification pour chaque bloc sont présentés dans le tableau 5.

\subsection{Validation externe de la cartographie}

La validation externe s'est appuyée sur 10854 points photo-interprétés sur des images SPOT. Le tableau 6 présente la matrice de confusion liée à cette validation. L'indice Kappa est de $79 \%$ et la précision globale se situe à $88 \%$. 


\begin{tabular}{|c|c|c|c|c|c|c|}
\hline Modèle testé & & 1 & 2 & 3 & 4 & 5 \\
\hline \multirow{3}{*}{$\begin{array}{c}\text { Variables } \\
\text { explicatives }\end{array}$} & Bandes spectrales & $1,2,3,4,5,7$ & $1,2,3,4,5,7$ & $1,2,3,4,5,7$ & $3,4,5,7$ & $3,4,5,7$ \\
\hline & $\begin{array}{l}\text { Indices d'occupation du } \\
\text { sol }\end{array}$ & & $\begin{array}{l}\text { ndvi, niri, } \\
\text { ndwi }\end{array}$ & & $\begin{array}{l}\text { ndvi,niri, } \\
\text { ndwi }\end{array}$ & $\begin{array}{l}\text { ndvi,niri, } \\
\text { ndwi }\end{array}$ \\
\hline & Indices morphologiques & & & $\begin{array}{l}\text { altitude, } \\
\text { rugosité, } \\
\text { pente }\end{array}$ & & $\begin{array}{c}\text { altitude, } \\
\text { rugosité, } \\
\text { pente }\end{array}$ \\
\hline \multirow{2}{*}{$\begin{array}{l}\text { Indices de } \\
\text { précisions du } \\
\text { modèle }\end{array}$} & OOB & $3,86 \%$ & $3,45 \%$ & $2,37 \%$ & $4,51 \%$ & $2,56 \%$ \\
\hline & Kappa & $94,77 \%$ & $95,33 \%$ & $96,80 \%$ & $93,89 \%$ & $96,54 \%$ \\
\hline
\end{tabular}

Tableau 4 : Qualité interne pour cinq modèles construits à partir de combinaisons de variables explicatives.

\begin{tabular}{cccccc}
\hline Indicateurs / blocs & Comatsa & Makira-Masoala & Caz & Cofav-Cofam & Fort Dauphin \\
\hline OOB & $2,90 \%$ & $2,30 \%$ & $3,61 \%$ & $1,39 \%$ & $2,15 \%$ \\
Kappa & $96,38 \%$ & $97,23 \%$ & $95,71 \%$ & $97,44 \%$ & $97,16 \%$ \\
$\begin{array}{c}\text { Nombre de parcelles } \\
\text { d'entrainement }\end{array}$ & 493 & 796 & 568 & 394 & 396 \\
\hline
\end{tabular}

Tableau 5: Validation interne du modèle de classification retenu (modèle 5): Indices de qualité pour chaque bloc d'étude.

\begin{tabular}{ccccccc}
\hline $\begin{array}{c}\text { Catégories de la carte } \\
\text { finale }\end{array}$ & FF & FP & PP & Total & $\begin{array}{c}\text { Erreur de } \\
\text { commission [\%] }\end{array}$ & $\begin{array}{c}\text { Précision } \\
\text { utilisateur [\%] }\end{array}$ \\
\hline FF & 5154 & 145 & 542 & 5841 & 12 & 88 \\
FP & 34 & 273 & 58 & 365 & 25 & 75 \\
PP & 372 & 53 & 4223 & 4648 & 9 & 91 \\
Total & 5560 & 471 & 4823 & 10854 & & \\
Précision producteur [\%] & 93 & 58 & 88 & & & \\
\hline
\end{tabular}

Tableau 6: Matrice de confusion externe pour la validation de la carte historique de déforestation pour la période $2010-$ 2013 (sur 10854 points de contrôle). FF : forêt en 2010 et 2013 ; FP : forêt en 2010 et prairie en 2013 ; PP : prairie en 2010 et 2013.

\subsection{Analyse de la déforestation et tendances}

La cartographie de l'évolution du couvert forestier est présentée dans la figure 5 . Le tableau 7 présente les superficies de la couverture de forêts naturelles et les taux de déforestation observés dans toute l'écorégion des forêts humides et dans deux strates d'altitude.

Ces résultats montrent que plus de 95000 ha de forêts par an ont été perdus pour la période 2005 - 2010 et environ 110000 ha par an pour la période 2010 2013.
Le taux annuel de déforestation pour l'écorégion des forêts humides a quasiment doublé entre les deux périodes : 2005 - 2010 et 2010 - 2013. La strate des forêts de basse altitude est visiblement la plus touchée par la déforestation depuis plusieurs années. Par ailleurs, la strate des forêts de moyenne altitude semble moins marquée par la déforestation. Néanmoins, les taux de déforestation y ont aussi doublé et y présentent aujourd'hui des valeurs assez proches de celles observées à basse altitude. 


\begin{tabular}{cccccc}
\hline \multirow{2}{*}{ Zone } & $\begin{array}{c}\text { Surface de la couverture forestière } \\
\text { (en milliers d'hectares) }\end{array}$ & \multicolumn{3}{c}{ Taux de déforestation (\% par an) } \\
& 2005 & 2010 & 2013 & $2005-2010$ & $2010-2013$ \\
\hline Toute l'écorégion & 4556 & 4457 & 4345 & 0,50 & 0,94 \\
Strate 0 - 800 & 1712 & 1666 & 1616 & 0,60 & 1,15 \\
Strate 800 et + & 2844 & 2790 & 2729 & 0,44 & 0,82 \\
\hline
\end{tabular}

Tableau 7: Superficies et taux annuels de déforestation (issus de la classification par Random Forest) par catégorie d'altitude pour les deux périodes observées : 2005 - 2010 et 2010 - 2013.

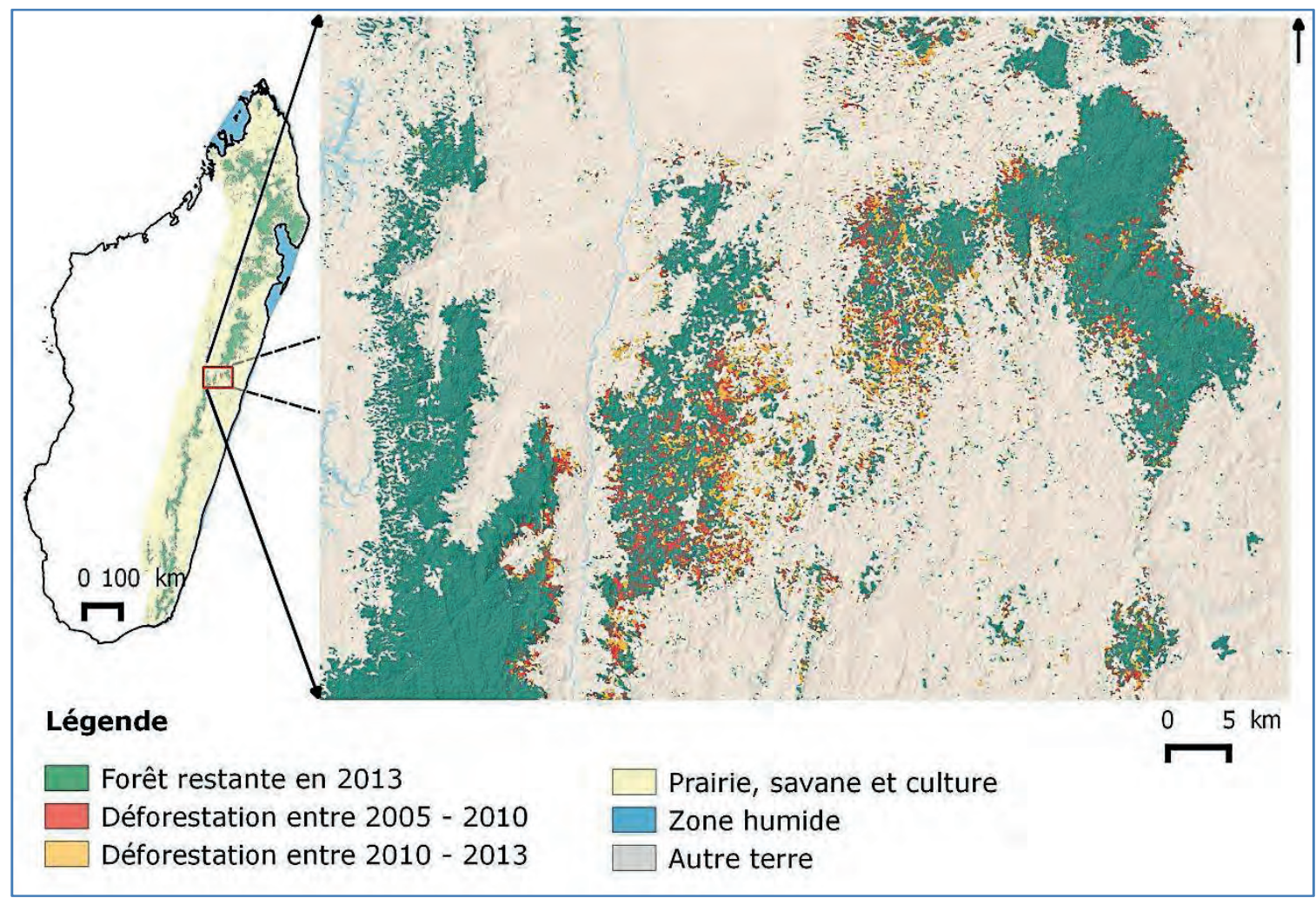

Figure 5: Carte de l'historique de la déforestation dans l'écorégion humide de l'Est de Madagascar avec un zoom sur une zone à forte intensité de déforestation (hot-spot)superposée sur une carte d'ombrage.

\section{Discussions}

\subsection{Paramétrage et qualité du modèle}

D'après Pontius et al., (2000), lorsque l'indice Kappa d'un modèle de classification est compris entre 0,50 et 0,75 le modèle peut être exploité. Les résultats de la validation interne que nous avons pour chaque bloc dépassent largement cette limite. Par ailleurs, Rodriguez-Galiano et al., (2012) suggèrent que l'indice OOB peut être considéré comme étant un indice de précision de classification non biaisé, mais il est compromis lorsque la précision globale de classification est faible $(<50 \%)$. Par contre, si la précision globale est particulièrement élevée (supérieure à 90\%), il est préférable de l'associer avec les indices Kappa dans l'évaluation d'un modèle.

En outre, il est clair que la qualité d'un modèle RF est très dépendante de la qualité des données d'entrainement (Gislason et al., 2006). C'est la raison pour laquelle les parcelles d'entrainement ont été construites en grappe dans cette étude pour que les frontières entre les typologies voisines soient bien délimitées (figure 2).Dans ce sens, il est important de noter que la photo-interprétation est dépendante de l'opérateur, de ce fait, il est préférable que deux photointerprètes s'en occupent pour minimiser les erreurs. Cette technique est fastidieuse, critique en ressource humaine et consommatrice de temps, mais nécessaire pour garantir la fiabilité des résultats.

Grinand et al., (2013) ont utilisé la combinaison de variable du modèle 4 . Pourtant, ce modèle n'est pas le plus optimal d'après nos résultats. Nous avons observé que le fait d'associer à ce modèle les indices morphologiques diminue l'erreur (modèle 5). Par ailleurs, d'autres méthodes de sélection de variables utilisant l'algorithme RandomForest ont été testées dans d'autres études comme dans Genuer et al., 2010. 


\subsection{Validation de la cartographie}

La précision globale de la classification est compatible avec d'autres études utilisant des images Landsat comme images de base pour la classification (Duveiller et al., 2008; Huang et al., 2009). Ces auteurs ont obtenu des précisions globales (PG) supérieures à $90 \%$. Nous avons obtenu ici une précision globale d'environ $90 \%$.La validation mise en œuvre dans cette étude est particulièrement importante puisque les points de références sont indépendants du résultat de classification. En effet, cette validation a été faite en utilisant des images SPOT - 5 qui sont différentes spatialement et spectralement aux images Landsat utilisées pour la classification. De plus, les opérateurs qui ont délimité les parcelles d'entrainement sont différents de ceux qui ont photo interprété les points de validation sur les images SPOT.

\subsection{Prise en compte de l'intervalle de temps dans le calcul du taux de déforestation}

Les taux de déforestation de chaque bloc ont été calculés en prenant en compte la surface forestière relative sur chaque scène (pondération). Des approches différentes peuvent être utilisées, notamment en pondérant par la surface de la scène concernée ou simplement en divisant par un intervalle de temps unique, moyen sur la zone. La comparaison de ces trois modes de calculs (tableau 8) montre des taux similaires dans les deux premiers cas et des résultats sensiblement différents lorsque l'on prend un intervalle de temps unique. Ces résultats confirment l'importance de considérer l'intervalle de temps dans le calcul de taux. Dans cette étude de déforestation historique, ainsi que dans d'autres sur Madagascar (MEFT-Cl, 2009 et MEFT-Cl-ONE, 2014), des écarts de plus d'une année entre deux scènes contigües sont observés du fait des contraintes de disponibilité des données exploitables en archive.

\subsection{Comparaison avec d'autres études historiques de déforestation}

Il est difficile de comparer les résultats de cette étude avec ceux des études similaires à l'échelle du pays puisque les méthodologies et les données utilisées ne sont pas compatibles (données, méthodologie, posttraitements). Toutefois, MEFT-Cl (2009) ont estimé que le taux de déforestation pour la période $2005-2010$ est à $0,5 \%$ par an pour les forêts humides. Ce taux est comparable avec ce qui est estimé dans cette étude (0,5\% par an). Pour la période $2010-2013$, le taux de la déforestation dans cette étude est proche du taux de la déforestation entre 1990 - 2000 (0,8\% par an) (MEFT-Cl, 2009), qui est également le taux maximum que le pays a connu depuis les dernières décennies.

\begin{tabular}{ccc}
\hline Méthode de calcul du taux de déforestation & \multicolumn{2}{c}{$\begin{array}{c}\text { Taux de déforestation } \\
\text { (\% de surface par an) } \\
2010-2013\end{array}$} \\
\hline Intervalle de temps unique pour toute la région & 0,23 & 0,92 \\
Intervalle pondéré par la surface de la scène & 0,26 & 0,91 \\
Intervalle pondéré par la surface forestière de la scène & 0,28 & 0,91 \\
\hline
\end{tabular}

Tableau 8: Comparaison des taux de déforestations dans le bloc Comatsa selon différents modes de calcul de l'intervalle de temps.

\section{Conclusions et perspectives}

Cette étude a permis d'actualiser les statistiques du couvert forestier et d'évaluer le rythme de la déforestation des forêts humides de l'Est de Madagascar. Elle met en lumière un taux de déforestation important $(0,5 \%$ par an entre 2005 2010 et $0,9 \%$ par an entre $2010-2013$ ) entre les deux périodes considérées, de nature à alerter toutes les parties prenantes œuvrant en faveur de la préservation des ressources naturelles et de la biodiversité à Madagascar. Couplée à un travail rigoureux de délimitation de parcelles d'entrainement par photointerprétation, cette étude permet de fournir une méthode de suivi de la dynamique forestière des forêts tropicales humides. L'ensemble des données et de la méthodologie mise en œuvre dans la présente étude permet de détecter des changements fins d'occupation du sol de manière fiable et d'envisager la réduction de la fréquence de suivi du couvert forestier à 3 ans. Cependant, si on veut réduire la fréquence de suivi de la déforestation et considérant des erreurs géométriques d'un pixel en moyenne, l'usage des images Landsat pourrait ne pas être satisfaisant compte tenu de sa résolution spatiale assez faible par rapport aux petites parcelles de déforestation à détecter. Afin d'augmenter la fréquence de suivi (à 1 ou 2 ans) tout en gardant la précision de la cartographie, le recours à des images de plus haute résolution spatiale (10 mètres) sera nécessaire. À ce titre, la disponibilité d'images de meilleures résolutions comme SPOT 6 ou Sentinel-2 pourra être très bénéfique pour l'analyse et le suivi de l'évolution de la couverture forestière à Madagascar. Ainsi, il serait possible de faire la détection de petites parcelles de tavy et maintenir une haute qualité des résultats. 


\section{Remerciements}

Cette étude a été menée dans le cadre du Projet EcoRégional REDD+ - Forêts Humides (PERR-FH) et a été financée via le Fonds Additionnel du Programme Environnemental III, et dans la continuité des travaux menés par le Programme Holistique de Conservation des Forêts (PHCF) financé par l'Agence Française de Développement et Air France. Les auteurs tiennent à remercier tous les membres du Consortium (WCS, ONE, MNP, Etc Terra) pour leur contribution dans la réalisation de cette étude.

\section{Références}

Achard, F., Eva, H., Stibig, H., J., Mayaux, P., Gallego, J., Richards, T., and Malingreau, J.P., 2002. Determination of deforestation rates of the world's humid tropical forests. Science, 297:pp 999-1003.

Breiman, L., 2001. Random Forests, Machine Learning, 45(1):pp 5-32

Breiman, L. 2002. Manual on setting up, using, and understanding random forests v3. 1. Technical Report, http://oz.berkeley.edu/users/breiman, Statistics Department University of California Berkeley, 29p.

Bylander, T. 2002. Estimating generalization error on two-class datasets using out-of-bag estimates. Machine Learning, 48: pp 287-297.

Caloz,R., and ColletC., 2001. Précis de Télédétection, Traitements numériques d'images de télédétection, vol. 3, Université de Québec, Agence Universitaire de la Francophonie. 385p

Cornet, A., 1974. Essai de cartographie bioclimatique à Madagascar. Notice explicative ORSTOM, 55: p 28.Paris, France.

Corbane, C., Lang, S., Pipkins, K., Alleaume, S., Deshayes, M., García Millán, V. E., and Michael, F., 2015. Remote sensing for mapping natural habitats and their conservation status - new opportunities and challenges. International Journal of Applied Earth Observation and Geoinformation, 37: pp 7-16. http://doi.org/http://dx.doi.org/10.1016/j.jag.2014.11.005

Cohen, J., 1960. A coefficient of agreement for nominales scales, Educational and Psychological Measurement, vol. 20, 1: pp $37-46$

DeFries, R., Asner, G. P., Achard, F., Justice, C. O.,LaPorte, N., Price, K., Small, C., and Towshend, J. 2005. Monitoring tropical deforestation for emerging carbon markets, P. Mountinho and S. Schwartzman, editors.Tropical Deforestation and Climate Change. IPAM and Environmental Defense, Belem, Brazil and Washington, DC, pp 35-44

Duveiller, G., Defourny, P., Desclée, B., and Mayaux, P.,2008. Deforestation in Central Africa: Estimates at regional, national and landscape levels by advanced processing of systematically-distributed Landsat extracts. Remote Sensing of Environment,112: pp 1969-1981.
Foody, G. M.,2010. Assessing the accuracy of land cover change with imperfect ground reference data. Remote Sensing of Environment, 114: pp 2271-2285.

Eastman, J.R., Sangermano, F., Ghimire, B., Zhu, H., Chen, H., Neeti, N., Cao, Y., and Crema, S., 2009. Seasonal Trend Analysis of Image Time Series, International Journal of Remote Sensing, 10:pp 27212726.

Fuller, R. M., Smith, G. M., and Devereux, B. J. 2003. The characterization and measurement of land cover change through remote sensing: Problems in operational applications? International Journal of Applied Earth Observation and Geoinformation, 4: pp 243-253.

Gao, B.C.,andGoetz,A.F.H., 1995. Retrieval of equivalentwater thickness and information related to biochemical components of vegetation canopies from AVIRIS data. Remote Sensing of Environment, 52:pp 155-162.

Genuer, R., Poggi, J.-M., and Tuleau-Malot, C. 2010. Variable selection using random forests. Pattern Recognition Letters, 31: pp 2225-2236

Gibbs, H.K., S. Brown, J. O., Niles, and Foley, J.A., 2007. Monitoring and Estimating Tropical Forest Carbon Stocks: Making REDD a Reality. Environmental Research Letters 2 (4): 045023.

GIEC, 2007 : Bilan 2007 des changements climatiques. Contribution des Groupes de travail I, II et III au quatrième Rapport d'évaluation du Groupe d'experts intergouvernemental sur l'évolution du climat. [Pachauri, R.K., Reisinger, A.,]. GIEC, Genève, Suisse, $103 p$.

Gislason, P. O., Benediktsson, J. A., and Sveinsson, J. R.,2006.Random forests for land cover classification. Pattern Recognition Letters, 27:pp 294-300.

Gutman, G., Byrnes, R., Masek, J., Covington, S., Justice, C., Franks, S., and Headley R. 2008.Towards monitoring land cover and land-use changes at a global scale. The Global Land Survey 2005, Photogrammetric Engineering and Remote Sensing, 74:pp 6-10,

Grinand, C., Rakotomalala, F., Gond, V., Vaudry, R., Bernoux, M., and Vieilledent, G. 2013.Estimating deforestation in tropical humid and dry forests in Madagascar from 2000 to 2010 using multi-date Landsat satellite images and the random forests classifier. Remote Sensing of Environment, 139: 68-80. http://dx.doi.org/10.1016/j.rse.2013.07.008.

GOFC-GOLD, 2014. A sourcebook of methods and procedures for monitoring and reporting anthropogenic greenhouse gas emissions and removals associated with deforestation, gains and losses of carbon stocks in forests remaining forests, and forestation. GOFC-GOLD Report version COP20-1, (GOFC-GOLD Land Cover Project Office, Wageningen University, The Netherlands).

Hansen, M. C., Potapov, P. V., Moore, R., Hancher, M., Turubanova, S. A.,Tyukavina, A., Thau, D., Stehman, S. V., Goetz, S. J., Loveland, T. R., 
Kommareddy, A., Egorov, A.,Chini, L., Justice, C. O., and Townshend, J. R. G. 2013. High-Resolution Global Maps of 21 st-Century Forest Cover Change. Science, 342: pp 850-53.

Harper, G., Steininger, M. K., Tucker, C. J., Juhn, D., and Hawkins, F. 2007. Fifty years of deforestation and forest fragmentation in Madagascar.Environmental Conservation, 34: pp 1-9.

Huang, C., Kim, S., Song, K., Townshend, J. R. G., Davis, P., and Alstatt, A.2009. Assessment of Paraguay's forest cover change using Landsat observations. Global and Planetary Change, 67:pp 112.

Humbert, H. 1965. Description des types de végétation In: HUMBERT, H. \& COURS-DARNE, G. (eds.). Notice de la carte de Madagascar. Travaux de Section Scientifique et Technique de I'Institut Français de Pondichéry. Institut Français de Pondichéry,6: pp 46-78.

IPCC, 2006. 2006 IPCC Guidelines for National Greenhouse Gas Inventories, Main, vol. 2, p. 12

Liaw, A., and Wiener, M., 2002. Classification and Regression by randomForest. R news, 2: pp 18 ?22.

Myers, N., Mittermeier, R.A., Mittermeier, C.G., da Fonseca, G.A.B., and Kent J. 2000. Biodiversity hotspots for conservation priorities.Nature, 403:pp 853858.

MEFT, Ministry of the Environment, Forest and Tourism of Madagascar, 2009. Evolution de la couverture de forêts naturelles à Madagascar, 19902000-2005. Antananarivo, Madagascar: USAID-CI, p132.

MEFT, ONE, Cl, 2013. Evolution de la couverture des forêts naturelles à Madagascar, 2000 - 2005 - 2010. ONE-CI-MEFT, p 11.

Menon, S., and Bawa, K.S., 1997. Application of geographic information systems, remote sensing, and landscape ecology approach to biodiversity conservation in Western Gharts. Curr.Sci, 75:pp 228 285

Narendra Prasad, S. 1998.Conservation planning for the Western Ghats of Kerala. II. Assessment of habitat loss and degradation. Curr. Sci, 75:pp 228 - 285.

Puyravaud, J. P. 2002. Standardizing the calculation of the annual rate of deforestation. Forest Ecology and Management, 177:pp 593-596.

Pontius, R.G.Jr. 2000. Quantification error versus location in comparison of categorical maps. Photogrammetric Engineering and Remote Sensing vol. 66, 8 :pp 1011-1016.

Rakotomalala, F. 2012. Evaluation de l'apport d'images à Très Haute Résolution Spatiale pour la modélisation de l'occupation du sol: cas du Complexe d'Aires Protégées de Zahamena. Mémoire de DEA, faculté des sciences, Université d'Antananarivo. p 20

Ramesh, B.R., Menon, S., and Bawa, K.S., 1997. A Vegetation besed approach to biodiversity gap analysis in the Agastyamalai region, Western Ghats, India. Ambio, 26: pp 536-539

Rodriguez-Galiano, V. F., Ghimire, B., Rogan, J., Chica-Oimo, M., and Rigol-Sanchez, J. P. 2012. An assessment of the effectiveness of a random forest classifier for land-cover classification. ISPRS Journal of Photogrammetry and Remote Sensing, 67: pp 93-104.

Rouse Jr., J. W., Haas, R. H., Schell, J. A., and Deering, D. W. 1973. Monitoring vegetation systems in the Great Plains with ERTS. In S. C. Freden, E. P. Mercanti, \& M. Becker (Eds.), Third Earth Resources Technology Satellite-1 Symposium. Technical presentations, section A, vol. I, pp. 309 317.Washington, DC: National Aeronautics and Space Administration (NASA SP-351).

RPP-Madagascar, 2014. Proposition des mesures pour l'état de préparation (Readiness - Preparation Proposal), [cabinet Soritra, ONF-International et le CT-REDD], version Juin 2014, p39.

Schneider, A. 2012. Monitoring land cover change in urban and peri-urban areas using dense time stacks of Landsat data and data mining approach. Remote Sensing of Environment, 124: pp 689-704.

Tachikawa, T., Manabu K., and Akira I. 2011. ASTER GDEM Version 2 Validation Report. In International Geoscience and Remote Sensing Symposium (IGARSS). pp. 1-24.

Teillet, P. M., Helder, D. L., Ruggles, T. A., Landry, R., Ahern, F. J., Higgs, N. J., and Palluconi, F. D. 2004. A definitive calibration record for the Landsat-5 thematic mapper anchored to the Landsat-7 radiometric scale. Canadian Journal of Remote Sensing, 30(4): pp 631643.

Tropek, R., Jan B., Petr K., Zuzana M., Irena, Š., and David S. 2014. Comment on "High-resolution global maps of 21st-centry forest cover change". Science $\begin{array}{lllll}\text { (New York, } & \text { N.Y.) } 344 & \text { (6187): } & \text { p } 981 .\end{array}$ doi:10.1126/science.1248753.

Verchot, L., and Petkova, E. 2009. The State of REDD Negotiations: Consensus Points, Options for Moving Forward and Research Needs to Support the Process. Bogor: CIFOR.pp 1-24.

Vieilledent, G., Grinand, C., and Vaudry, R. 2013. Forecasting deforestation and carbon emissions in tropical developing countries facing demographic expansion: a case study in Madagascar. Ecology and Evolution, 3(6):pp 702-1 716

Williams, M. 2002. Deforesting the Earth: From Prehistory to Global Crisis. Annals of the Association of American Geographers, 96(3): pp 674-75.

White, F., 1983. The vegetation of Africa, a descriptive memoir to accompany the UNESCO/AETFAT/UNSO vegetation map of Africa. UNESCO, Natural Resource. Res, 20: pp 1-356 